\title{
EXPONENTIAL ACTION OF A PENDULUM
}

\author{
BY R. E. MEYER ${ }^{1}$
}

\author{
Communicated by George Carrier, May 15, 1973
}

\begin{abstract}
The total change in the action of a slowly modulated oscillator is transcendentally small in the slowness parameter, if the modulation is smooth. To approximate it thus requires methods for bypassing the whole asymptotic expansion of the oscillator. A theorem on the exponential property of the action for analytic frequency variation and an outline of an elementary proof are reported. It rests on an asymptotic split of the angle variable into its algebraic and exponential parts.
\end{abstract}

1. Introduction. The amplitude of a pendulum of slowly changing length has an asymptotic expansion in the small parameter $\varepsilon$, but the action is an 'adiabatic invariant': its total change has the zero expansion, if the length changes smoothly enough. A host of asymptotic methods have been developed for such slowly modulated oscillators-stationary phase, WKB, two-timing, etc.-but all lead to asymptotic expansions and are helpless when an approximation to a transcendental property is needed.

To promote development of methods for bypassing expansions to strike directly at subdominant properties, we report an extension of the theorem of Knorr and Pfirsch [1] on the exponential property for analytically smooth frequency and outline an elementary proof. An elementary proof of the transcendental property [1], [2], [3], [4] falls out as a bonus.

The method rests on an asymptotic split of the angle variable into its algebraic and exponential parts and on a Fourier representation of the action change with respect to an intrinsic pendulum time.

A precise order-estimate for the action change is reported for a class of frequency functions of practical interest (Theorem 2).

2. Main results. Let $q(t ; \varepsilon)$ be a solution of $d^{2} q / d t^{2}+\omega^{2} q=0$ with $\omega=\omega(\tau), \tau=\varepsilon t, 0<\varepsilon<1$, and let $2 P(t)=\omega q^{2}+\omega^{-1}(d q / d t)^{2}$. The following hypotheses will be distinguished.

AMS (MOS) subject classifications (1970). Primary 41A60, 70J20; Secondary 70H99, 70-00, 76X05.

Key words and phrases. Asymptotics, adiabatic invariance.

${ }^{1}$ Supported in part by NSF grant GP-33579X, the Wisconsin Alumni Research Foundation, and the British Science Research Council. 
(A) For $\tau \in \boldsymbol{R}, \omega \in \boldsymbol{R}$ with glb $\omega>0, \omega^{\prime} \in L(\boldsymbol{R})$ and $\omega$ tends to limits as $\tau \rightarrow \pm \infty$.

(B) For $\tau \in \mathbf{C}, \omega$ is analytic on a strip $\Delta$ of positive minimal width about the real axis and $\omega^{\prime} \rightarrow 0$ as $\mid$ re $\tau \mid \rightarrow \infty$ in $\Delta$ locally uniformly in im $\tau$.

$\left(\mathbf{B}^{\prime}\right)$ For $\tau \in \mathbf{C}, \omega^{2}$ is analytic on a neighborhood of the real axis in which re $\omega>0$ at all re $\tau$ for

$$
0<\operatorname{im} \int_{0}^{r} \omega(s) d s<\text { const }=m
$$

and in which $\omega^{2}$ has a root at $\operatorname{im} \int \omega=m>0$; and $\omega^{\prime} \rightarrow 0$ as $\mid$ re $\tau \mid \rightarrow \infty$ locally uniformly in im $\tau$.

THEOREM 1. Under hypotheses (A), (B), $\exists d>0$ such that

$$
\lim _{t \rightarrow \infty} \frac{P(t)-P(-t)}{P(-t)}=O\left(e^{-c / \varepsilon}\right)
$$

as $\varepsilon \rightarrow 0$ for all $c<d$.

Theorem 2. Under hypotheses (A), ( $\left.\mathrm{B}^{\prime}\right)$,

$$
\lim _{t \rightarrow \infty} \frac{P(t)-P(-t)}{P(-t)}=O\left(e^{-2 m / \varepsilon}\right) .
$$

Examples satisfying (A) and $\left(\mathrm{B}^{\prime}\right)$ are $\omega(\tau)=M-(M-1) \exp \left(-\tau^{2}\right)$ with $M=$ const $>1$ [5] for which $m=M \mu+\frac{1}{2} i \pi^{1 / 2}(M-1) \operatorname{erf}(i \mu), \mu^{2}=$ $\log [M /(M-1)]$, and $\omega=1+\frac{1}{3} \tanh \tau$ [6] for which $m=\pi / 3$, and $\omega^{2}=$ $1+\left(1+2 e^{-\tau}\right)^{-1}[4]$ for which $m=\pi$.

3. Rotation at real time. $F(q, Q, t ; \varepsilon)=\frac{1}{2} q^{2} \omega \cot Q$ generates a canonical transformation [7] to variables $P, Q$ of action-angle type and Hamiltonian equations

$$
\frac{d Q}{d t}=\omega+\frac{d \omega / d t}{2 \omega} \sin 2 Q, \quad \frac{d P}{d t}=-P \frac{d \omega / d t}{\omega} \cos 2 Q
$$

whence

$$
\log \frac{P(\infty)}{P(-\infty)}=-\operatorname{re} \int_{-\infty}^{\infty} \frac{\omega^{\prime}}{\omega} r d \tau=-\operatorname{re} \Pi(\varepsilon)
$$

and the 'rotation' $\exp (2 i Q(t ; \varepsilon))=r(z)$ satisfies

$$
r^{\prime}(z)=i \varepsilon^{-1} r+\phi\left(r^{2}-1\right), \quad 2 \phi(z)=\Omega^{\prime} / \Omega
$$

in terms of

$$
z(\tau)=2 \int_{0}^{\tau} \omega(\sigma) d \sigma, \quad \Omega(z)=\omega(\tau) .
$$


A frequency hypothesis of type (A) is now seen to be necessary for the pendulum to have classically defined actions as $|t| \rightarrow \infty$ and real-valued total action change.

LEMMA 1. If $\phi(z) \in L(\boldsymbol{R}) \cap C(\boldsymbol{R})$ and is real-valued, $0<\varepsilon<1$, and $A(z ; \alpha)$ satisfies $(3)$ and $A(\alpha ; \alpha)=0$, then

for some $\alpha \in \overline{\boldsymbol{R}}$.

$$
I(\alpha)=2 \int_{-\infty}^{\infty} \phi(z) \operatorname{re} A(z ; \alpha) d z=0
$$

With this choice of $\alpha$, it follows that

$$
\Pi(\varepsilon)=2 \rho(\alpha) \int_{-\infty}^{\infty} \phi(z) k(z) e^{i z / \varepsilon} d z
$$

where $\rho(\alpha)=r(\alpha) \exp (-i \alpha / \varepsilon),|\rho|=1$, and

$$
\begin{aligned}
k(z) & =[r(z)-A(z ; \alpha)] e^{i(\alpha-z) / \varepsilon} / r(\alpha) \\
& =h(z) /\left[1-r(\alpha) \int_{\alpha}^{z} \phi h e^{i(s-\alpha) / \varepsilon} d s\right]
\end{aligned}
$$

by (3), with

$$
h(z)=\exp \left(2 \int_{\alpha}^{z} \phi(s) A(s ; \alpha) d s\right) .
$$

LemMA 2. Under hypothesis (A), $\operatorname{lub}_{z \in \mathbb{R}}|A| \rightarrow 0$ as $\varepsilon \rightarrow 0$.

CoROllaRy 2a. $\quad \lim _{\varepsilon \rightarrow 0} k(z) \equiv 1$ uniformly in $z$.

The action change is represented by (2), (5) as a Fourier transform from the asymptotic theory of which [8], [9] we deduce

THEOREM 3. Transcendental smallness of the total action change requires frequency $\omega(\tau) \in C^{\infty}(\boldsymbol{R})$; exponential smallness (in the strict sense of Theorem 1) requires analyticity of $\omega(\tau)$.

4. Analytic frequency. By (A), (B) and (4), $\Omega(z)$ is also analytic on a strip about the real axis of minimal width, say, $d>0$. By (3), (4), (6), (7), $r(z), A(z ; \alpha), h(z)$ and $k(z)$ are analytic on this strip.

Lemma 3. Under hypotheses (A), (B), Lemmas 2, 2a hold on any closed subset of $\{0 \leqq \operatorname{im} z<d\}$.

It follows from (6) that $r-A$ is then exponentially small; conversely, $A(z ; \alpha)$ must contain any algebraic part (as $\varepsilon \rightarrow 0)$ of $r(z)$. Hypothesis (B) 
permits us to shift the path in (5) to fixed im $z>0$, whence Theorem 1 follows.

Hypothesis $\left(B^{\prime}\right)$ permits us to shift the path to a fixed im $z>2 m$, except for loops around the branch points $z_{k}$ with im $z_{k}=2 m$. Let $D$ denote the domain bounded by this path and the real axis. The loops make independent contributions, and by comparison, the rest of the path makes only an exponentially small contribution. For Theorem 2, it therefore suffices to consider

$$
\Gamma(\varepsilon)=e^{-i z_{0} / \varepsilon} \Pi(\varepsilon) / \rho=2 \int_{L} \phi(z) k(z) e^{i\left(z-z_{0}\right) / \varepsilon} d z
$$

over a loop $L$ around a point $z_{0}=2 \int_{0}^{\tau_{0}} \omega(\sigma) d \sigma$ at which $\omega(\tau)$ has an expansion

$$
\omega(\tau)=\omega_{0}\left(\tau-\tau_{0}\right)^{v / 2}\left(1+\sum_{1}^{\infty} \omega_{n}\left(\tau-\tau_{0}\right)^{n}\right)
$$

with integer $v \geqq 1$ and $\omega_{0}=$ const $\neq 0$. By (6), (7), evaluation of (8) requires information on the special rotation $A(z ; \alpha)$ near such a singular point of (3).

LEMMA 4. If $p=d q / d t, d p / d t=-\omega^{2} q$ with $i p(\tau(\alpha) / \varepsilon)=\omega q(\tau(\alpha) / \varepsilon) \neq 0$ (where $\tau(z)$ denotes the inverse of (4) on $\boldsymbol{R})$, then $(i p-\omega q) /(i p+\omega q)=$ $A(z ; \alpha)$ on $D$.

Thus $\tau_{0}$ is a turning point of the differential equation of the pendulum. By the help of a comparison equation, its Stokes multipliers [10] and extension of Lemma 3 to any closed subset of $D, \lim _{\varepsilon \rightarrow 0} \Gamma$ can finally be represented as a product of two convergent integrals computable purely locally from (3) on an $\varepsilon$-neighborhood of $z_{0}$.

Details will appear in ZAMP.

\section{REFERENCES}

1. G. Knorr and D. Pfirsch, The variation of the adiabatic invariant of the harmonic oscillator, Z. Naturf. 21a (1966), 688-693.

2. C. S. Gardner, Adiabatic invariants of periodic classical systems, Phys. Rev. (2) 115 (1959), 791-794. MR 21 \#7715.

3. J. E. Littlewood, Lorentz's pendulum problem, Ann. Physics 21 (1963), 233-242. MR 27 \#6419.

4. W. Wasow, Adiabatic invariance of a simple oscillator, SIAM J. Math. Anal. 4 (1973), 78-87.

5. J. E. Howard, Nonadiabatic harmonic oscillator, Phys. Fluids 13 (1970), 24072412.

6. F. Hertweck and A. Schlüter, Die "adiabatische invarianz" des magnetischen bahnmomentes geladener teilchen, Z. Naturf. 12a (1957), 844-849. MR 20 \#630. 
7. H. Goldstein, Classical mechanics, Addison-Wesley, Cambridge, Mass., 1951. MR 13, 291.

8. E. C. Titchmarsh, Introduction to the theory of Fourier integrals, Clarendon Press, Oxford, 1937.

9. D. S. Jones, Fourier transforms and the method of stationary phase, J. Inst. Math. Appl. 2 (1966), 197-222. MR 34 \#4820.

10. H. L. Turrittin, Stokes multipliers for asymptotic solutions of a certain differential equation, Trans. Amer. Math. Soc. 68 (1950), 304-329. MR 11, 595.

Fluid Mechanics Research Institute, University of EsSex, EsSex, England

Department of Mathematics, University of Wisconsin, Madison, Wisconsin 53706 